\title{
Elastic and Plastic Strain Measurement Using Electron Backscatter Diffraction Technique: The Influence of Sample Preparation
}

\author{
Pawel Nowakowski ${ }^{*}$, Jorg Wiezorek ${ }^{2}$, Ian Spinelli ${ }^{3}$, Mary Ray ${ }^{1}$ and Paul Fischione ${ }^{1}$ \\ 1. E.A. Fischione Instruments, Inc., Export, PA, USA. \\ 2. University of Pittsburgh, Pittsburgh, PA, USA. \\ 3. GE Global Research Center, Niskayuna, NY, USA. \\ *Corresponding author: p_nowakowski@fischione.com
}

In materials science, knowledge about elastic and plastic strain, as well as their distribution in crystalline materials, is critical when studying phenomena such as stress fatigue, corrosion cracking, and formability. In semiconductor devices, elastic and plastic strain can introduce crystallographic defects and cause fracture and delamination, which can dramatically change electrical properties and device reliability. For these reasons, the measurement of plastic and elastic strain was widespread in industrial and academic environments in the past decade. Many techniques have been developed and used for plastic and elastic strain study [1-5]. In microscopy and microanalyses, one of the techniques used for deformation structures characterization is electron backscatter diffraction (EBSD). The EBSD plastic strain measurement techniques rely on recording changes in EBSD pattern quality [6,7] or local changes of crystal orientation within a grain $[8,9]$. Recently, the EBSD technique was applied to elastic strain measurements. This approach is based on cross-correlation analysis of high resolution EBSD patterns. The technique measures lattice distortion-related differences between EBSD patterns obtained from the same grain and it is known as HR-EBSD [10-13]. Both strain studies by EBSD and HR-EBSD are very sensitive to diffraction pattern quality. Aside from intrinsic instrumentation limitations, sample preparation factors prominently into accuracy and precision attained in HR-EBSD strain analyses. Surface contamination, oxidation, crystal lattice damage, and plastic deformation must be avoided during sample preparation to obtain accurate and precise measurements.

Here we report on elastic strain measurements associated with the $\left(\gamma / \gamma^{\prime}\right)$ - interfaces in Ni-superalloy by HR-EBSD. We also present plastic strain measurements in titanium alloys and aluminum alloys by conventional EBSD. We report on the influence of two samples preparation methods in HR-EBSD strain mapping for crystalline materials: (i) conventional mechanical polishing (MP) using colloidal silica suspension (Figure 1a, 2a), and (ii) Ar broad ion beam (BIB) milling using low ion energy and milling angle (Figure 1b, 2b). When comparing the MP and BIB methods, the analyses revealed:

- Average elastic strain magnitudes for the principal strain components differ by up to two orders of magnitude; higher for MP samples and lower for BIB milled samples.

- Plastic strain measured in terms of local crystal orientation gradient changes can be $\sim 35 \%$ higher for MP samples, as compared to BIB milled samples.

This could be attributable to the introduction of dislocations by plastic deformation stemming from local interactions with abrasives under the associated complex mechanical loading geometries during MP. The density and distribution of geometrically necessary dislocations can be determined by HR-EBSD and will be discussed. 
References:

[1] L Margulies, G Winther and HF Poulsen, Science 23 (2001), p. 2392.

[2] AJ Wilkinson and DJ Dingley, Acta Metallurgica et Materialia 39 (1991), p. 3047.

[3] S Kaboli et al., Microscopy and Microanalysis 19 (2013), p. 1620.

[4] MD Vaudin et al., Ultramicroscopy 111 (2011), p. 1206.

[5] JQ Da Fonseca, PM Mummery and PJ Withers, Journal of Microscopy 218 (2005), p. 9.

[6] AJ Wilkinson, G Gonzalez and DJ Dingley, Journal of Microscopy 169 (1993), p. 255.

[7] M Kamaya, AJ Wilkinson and JM Titchmarsh, Nuclear Engineering and Design 235 (2005), p. 713.

[8] EM Lehockey, Y-P Lin and O Lepik in "Electron Backscatter Diffraction in Materials Science," eds.

AJ Schwartz, M Kumar, and BL Adams (Springer, Boston) p. 247.

[9] R Ørsund, J Hjelen and E. Nes, Scripta Metallurgica 23 (1989), p. 1193.

[10] KZ Troost, P van der Sluis and DJ Gravesteijn, Applied Physics Letters 62 (1993), p. 1110.

[11] AJ Wilkinson, Ultramicroscopy 62 (1996) p. 237.

[12] AJ Wilkinson, G Meaden and DJ Dingley, Ultramicroscopy 106 (2006), p. 307.

[13] TB Britton and AJ Wilkinson, Ultramicroscopy 111 (2011), p. 1395.
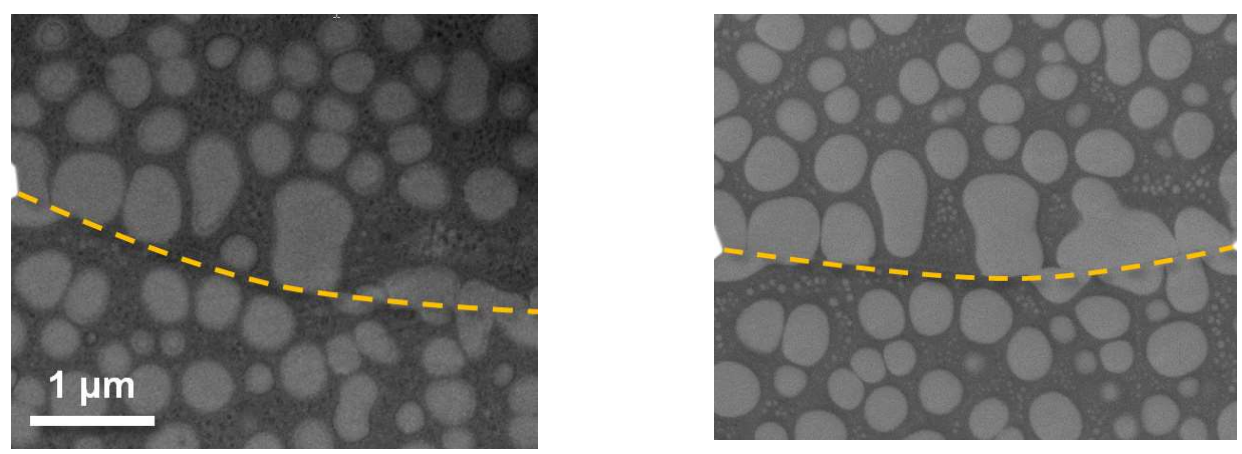

Figure 1. SEM image of Ni-superalloy surface after mechanical polishing (left) and after broad ion beam Ar ion milling (right); the yellow line marks the grain boundary.
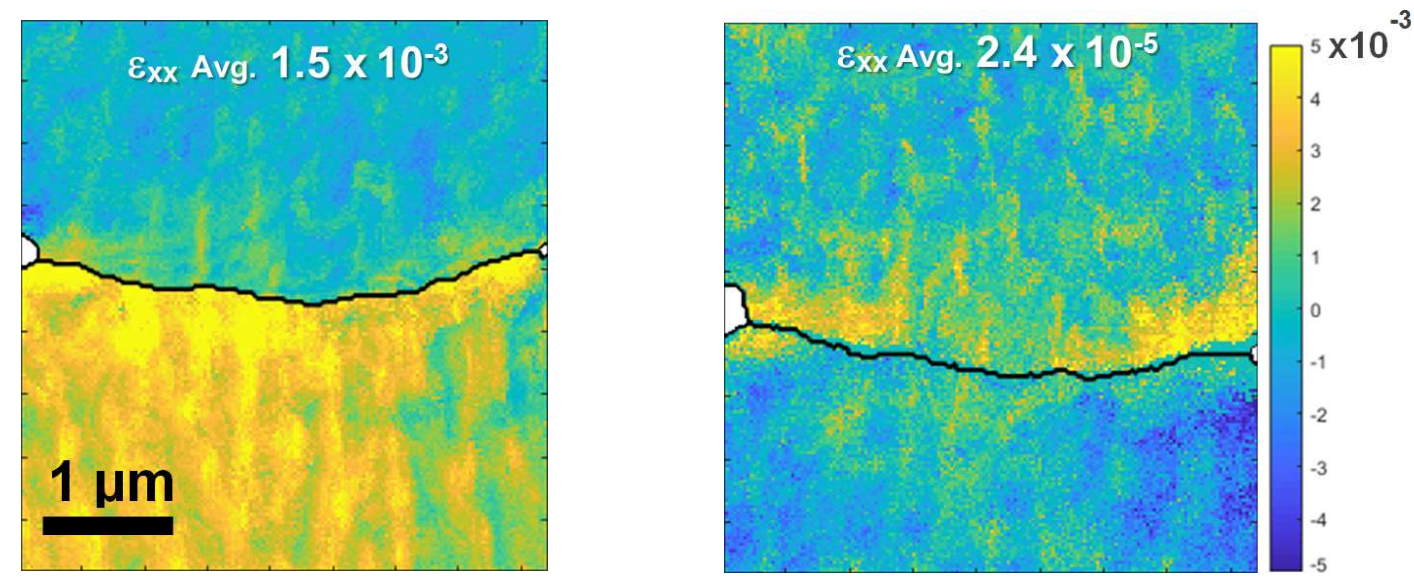

Figure 2. HR-EBSD elastic strain maps (collected from the same area shown in Figure 1): after mechanical polishing (left) and after broad ion beam Ar ion milling (right). 